\title{
Editorial note to: Karl Stellmacher, On the initial value problem of the equations of gravitation
}

\section{Helmut Friedrich}

Published online: 21 April 2010

(C) Springer Science+Business Media, LLC 2010

Keywords Solutions of Einstein's equations · Initial value problem for Einstein's equations in vacuum and with a dust source $\cdot$ Golden Oldie

The general relativistic notion of 'domain of dependence' is important in the (global) analysis of gravitational fields. It is technically convenient because it is a geometrical concept defined, irrespective of any field equations, purely in terms of the metric. Its fundamental importance, however, comes from the fact that it gives precise meaning to the statement that the evolution process of the Einstein equations respects the notion of causality defined by the solution metrics of these equations.

This is taken for granted nowadays but it was not obvious in the early days of General Relativity. It is the merit of Stellmacher's article that it clarifies this issue for the first time. Previous work by A. Einstein, D. Hilbert, G. Darmois, C. Lanczos and others, which revealed important properties of the field equations, was based on the study of linearizations, formal expansions and CauchyKowalevskaja type arguments. These techniques did not allow one to draw any conclusion of the type given in Stellmacher's article. The new ingredient he uses is an argument based on 'energy integrals' which was proposed by K. O. Friedrichs and H. Lewy (cf. [1] for earlier work in this direction). It is discussed in section 2 (we note that the inequality following equations (3) is slightly misleading and should be replaced by the statement that the mean value theorem is used to obtain these equations).

The republication of the original paper can be found in this issue following the editorial note and online via doi:10.1007/s10714-010-0960-1.

H. Friedrich ( $\otimes)$

Max-Planck-Institut für Gravitationsphysik, Am Mühlenberg 1, 14476 Golm, Germany

e-mail: hef@aei.mpg.de 
It appears to be the first time that this powerful argument has been used in the context of general relativity. It has largely been generalized by now and 'energy estimates' represent an indispensable tool in the existence theory.

Stellmacher analyses the Einstein-Maxwell equations in harmonic and Lorentz gauge respectively, arguing that these gauge conditions themselves respect the causality relations defined by the solution metric. In this case the proof that the domains of (geometric) uniqueness for the field equations coincide with the domains of dependence defined by the metric is short, clear, and clean. He also analyses the case of pressure free matter (dust) which is more complicated and his results are in fact not so clear and complete because they only refer to regions where neither caustics nor matter-vacuum interfaces occur. This should not be held against him, the situation is complicated and poses problems even nowadays (cf. the discussion in [2]). He finally also remarks that he did not succeed in the case of more general matter fields. Again, this is not surprising.

While Stellmacher's results and methods are standard now they were certainly new at the time and marked in a sense a kind of 'phase transition' in the analysis of the field equations. Had the times been better (K. O. Friedrichs who had suggested the work had left the country for political reasons half a year before the article was submitted) it might have led to a much earlier clarification of the local Cauchy problem.

\section{Karl Ludwig Stellmacher—a brief biography}

By Hubert Goenner, based on Ref. [3].

Karl Ludwig Stellmacher (1910-2001) studied mathematics, physics and chemistry at the University of Göttingen from 1927-1933 in order to become a teacher at a Gymnasium (high school/first two college years). His final exam did not recommend him for a PhD. Nevertheless, Richard Courant seems to have given him a problem in hyperbolic differential equations. After having lost Courant as an advisor, in 1935 the mathematician Gustav Herglotz (1881-1953) took him on and proposed propagation of gravity as a subject. Stellmacher finished his dissertation in 1937; it is the paper reproduced here. Surprisingly, he credited Kurt Friedrichs as responsible for the theme who, in the same year left his position in Braunschweig/ Germany as a consequence of his political views and his jewish fiancée (whom he later married).

After his dissertation, Stellmacher worked as an assistant to Max Schuler (18821972), director and successor of the famous pioneer of aerodynamics, Ludwig Prandtl (1875-1853), in the Kaiser-Wilhelm Institute for Fluid Dynamics, on problems concerning gyroscopes. During the 2nd world war, he had to be a soldier from 1939 to 1944. After the war, his results on the gyroscope found no interest such that he went back to pure mathematics.

In 1948 he became both an assistant and a Privatdozent (lecturer) in the Mathematics Institute of the University of Göttingen. After having received the title of professor (but not the pay) in 1955, he left Göttingen immediately to become a research associate in the Institute for Fluid Dynamics and Applied Mathematics of the University of 
Maryland in College Park. Within a year, he became full professor and stayed there until retirement in 1977.

\section{References}

1. Courant, R., Hilbert, D.: Methods of Mathematical Physics II. Interscience, New York (1962)

2. Choquet-Bruhat, Y., Friedrich, H.: Class. Quantum Grav. 23, 5941 (2006)

3. Rammer, G.: Die Nazifizierung und Entnazifizierung der Physik an der Universität Göttingen, p. 512. Dissertation, Göttingen, 2004 